\title{
Acquired resistance to osimertinib in patients with non-small-cell lung cancer: mechanisms and clinical outcomes
}

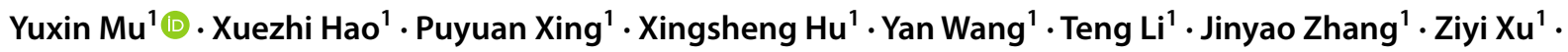 \\ Junling $\mathrm{Li}^{1}{ }^{10}$
}

Received: 9 January 2020 / Accepted: 27 April 2020 / Published online: 8 May 2020

(c) The Author(s) 2020

\begin{abstract}
Purpose Osimertinib, a third-generation epidermal growth factor receptor tyrosine-kinase inhibitor (EGFR-TKI), has demonstrated substantial clinical benefit in patients with non-small-cell lung cancer (NSCLC) who were resistant to early-generation EGFR-TKIs and had acquired a T790M mutation. The aim of our study was to identify the mechanisms underlying resistance to osimertinib and to correlate them with clinical outcomes.

Methods We retrospectively analyzed patients with advanced NSCLC who received osimertinib for T790M-mutated acquired resistance to prior EGFR-TKIs between March 1, 2017 and December 31, 2018. Patients with paired molecular data of preosimertinib and after resistance development, which were not confirmed with small-cell lung cancer (SCLC) transformation, were included in the molecular analysis set.

Results Of 49 patients evaluated in the molecular analysis set, 24 patients maintained T790M mutation, while 25 patients exhibited T790M-loss. Molecular modifications were identified in 27 of 49 patients including EGFR acquired mutations (C797S, C796S, G796S, V802I, V834L, E758D and G724S), non-EGFR-dependent mutations (PIK3CA, ALK, BRAF, KRAS and TP53), EGFR amplification and MET amplification. At data cutoff, median progression-free survival (PFS) was 9.3 months in the T790M-retain group compared with 7.8 months in T790M-loss patients $(P=0.053)$. Median PFS was significantly longer in patients with EGFR-dependent resistance mechanism (13.5 months) than in those with alternative pathway activation ( 8.2 months; $P=0.012$ ).

Conclusions The study revealed heterogeneous mechanisms of resistance to osimertinib in advanced NSCLC patients and their association with clinical outcomes. Patients who maintained T790M mutation or with EGFR-dependent resistance mechanism had longer clinical outcome benefits.
\end{abstract}

Keywords Non-small-cell lung cancer $\cdot$ Osimertinib $\cdot$ T790M $\cdot$ Resistance mechanism

\section{Introduction}

Lung cancer is the leading cause of cancer-related deaths worldwide (Siegel et al. 2015). The discovery of smallmolecule anti-cancer drugs, which target specific oncogenic driver mutations, dramatically changed the clinical therapeutic modality for patients with non-small-cell lung cancers

Junling Li

13520990822@163.com; lijunling@ cicams.ac.cn

1 Department of Medical Oncology, National Cancer Center/ National Clinical Research Center for Cancer/Cancer Hospital, Chinese Academy of Medical Sciences and Peking Union Medical College, Number 17 Panjiayuan Nan Li, Chao Yang District, Beijing 100021, China
(NSCLC). The most common oncogenic driver mutation in lung cancer is epidermal growth factor receptor (EGFR) mutation, which characterizes approximately $40-55 \%$ of all NSCLC in Asian patients (Shi et al. 2014). Numerous randomized trials have demonstrated the superiority of smallmolecule EGFR tyrosine-kinase inhibitors (TKIs) over cytotoxic chemotherapy for the treatment of EGFR-mutant NSCLC patients and, thus, have been established as standard first-line therapy (Mok et al. 2009; Rosell et al. 2012; Sequist et al. 2013). Most patients who were treated with first- or second-generation EGFR-TKIs developed acquired resistance after about 8-14 months, and approximately 50-60\% of patients had a T790M acquired resistance mutation (Yu et al. 2013; Lee et al. 2019). Osimertinib is a potent, irreversible, third-generation EGFR-TKI, which inhibits both 
EGFR-activating and T790M resistance mutations (Cross et al. 2014). The AURA3 phase III trial showed that osimertinib had a superior performance to standard platinum-based chemotherapy in terms of the objective response rate (ORR), progression-free survival (PFS) and tolerability in patients with T790M-mediated acquired resistance (Mok et al. 2017). Osimertinib has been approved for the treatment of NSCLC patients carrying a T790M resistance mutation after disease progression of prior EGFR-TKI therapy. Various resistance mechanisms of osimertinib have been reported including EGFR modifications, alternative pathway activation or histological transformation, but these are not yet fully understood (Oxnard et al. 2018). The prognostic significance of different resistance mechanisms has not been well documented in the literature. The aim of our study was to elucidate the mechanisms of acquired resistance to osimertinib and to explore the association of clinical outcomes with various genetic modifications.

\section{Materials and methods}

\section{Study population}

Between March 1, 2017 and December 31, 2018, patients who received osimertinib were retrospectively identified in our cancer center. Inclusion criteria included histologically or cytologically confirmed NSCLC, advanced stage (including stages IIIB and IV), acquired resistance to prior EGFRTKI therapy and harboring T790M resistance mutation in either tumor or plasma samples before receiving singleagent osimertinib therapy. Patients who received osimertinib for less than 3 weeks for any reason were excluded from the study. Patients with paired molecular information before osimertinib initiation and after progression, and who were not confirmed with small-cell lung cancer (SCLC) transformation were included in the molecular analysis set. A flowchart outlining the selection of 49 patients in the molecular analysis set is presented in Fig. 1. We retrospectively collected demographic and clinical characteristics of patients including sex, age, smoking history, histology, Eastern Cooperative Oncology Group performance status (ECOG PS), treatment history, metastases sites prior to osimertinib, osimertinib treatment procedure and molecular information. The data cutoff for analysis was May 14, 2019.

\section{Assessments}

The primary aim of the study was to characterize the mechanisms of resistance to osimertinib in patients previously treated for NSCLC. Plasma and tumor samples of pre-osimertinib and after radiological identified progression were mostly tested using next-generation sequencing (NGS)

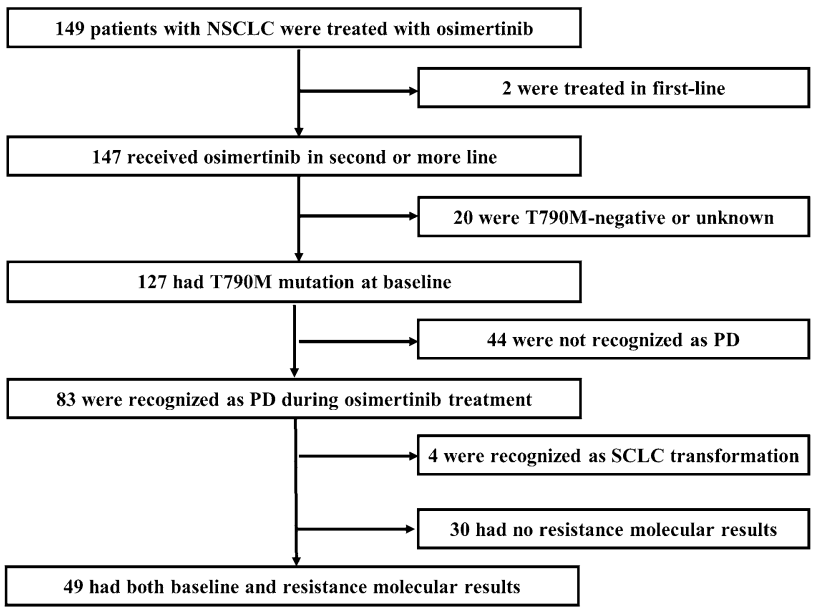

Fig. 1 Flowchart of patient selection in the molecular analysis set. NSCLC non-small-cell lung cancer, $P D$ progressive disease, SCLC small-cell lung cancer

panels (168-gene panel), according to their medical records, and paired molecular results were analyzed. The clinical aim was to explore the association of clinical outcomes with various genetic modifications. Clinical response was investigated according to Response Evaluation Criteria in Solid Tumors (RECIST) version 1.1. Disease control rate (DCR) was defined as the percentage of patients who had complete response (CR), partial response (PR) or stable disease (SD). PFS was defined as the duration from the start of osimertinib therapy until progressive disease (PD) or death from any cause. The treatment duration (TD) was measured from the start of osimertinib until the last dose for any reason.

\section{Statistical analysis}

Statistical analyses were carried out using SPSS 23.0 statistical software (SPSS, Inc., Chicago, IL, US). Survival analyses were performed using the Kaplan-Meier method and compared using a log-rank test between groups. A chisquared test and Fisher's exact test were used for comparing DCR. All $P$ values were two sided and a $P$ value $<0.05$ was considered to be statistically significant.

\section{Results}

\section{Patients and characteristics}

We collected baseline molecular data from 53 patients treated with osimertinib who had acquired a T790M resistance mutation to first- or second-generation EGFR-TKIs. All patients were adenocarcinomas at baseline and received osimertinib $80 \mathrm{mg}$ once daily. After resistance to osimertinib became apparent, 21 patients underwent re-biopsies, 
in which 4 patients were confirmed with a SCLC transformation. Forty-nine patients who were not confirmed with a histology transformation, and with paired molecular data of pre-osimertinib and after osimertinib resistance, were included in the molecular analysis set. Patient demographics and baseline characteristics of the molecular analysis set are presented in Table 1. Twenty-five patients received osimertinib as second line, the other 24 patients were third or later line. Median age was 59 years, $61.2 \%$ of patients were women, $77.6 \%$ were non-smokers and $95.9 \%$ had an ECOG PS of 0 or 1. EGFR-T790M/19del-positive and $\mathrm{T} 790 \mathrm{M} / \mathrm{L} 858 \mathrm{R}$-positive were detected in $30(61.2 \%)$ and 17 (34.7\%) patients at baseline, respectively. Prior to osimertinib, EGFR-TKI treatment history included gefitinib (25/49, $51.0 \%)$, erlotinib $(10 / 49,20.4 \%)$, icotinib $(18 / 49,36.7 \%)$, afatinib $(1 / 49,2.0 \%)$ and avitinib (3/49, 6.1\%). Lung (38/49, $77.6 \%)$, bone $(28 / 49,57.1 \%)$, pleural $(19 / 49,38.8 \%)$ and central nervous system (CNS) $(18 / 49,36.7 \%)$ metastases were common metastatic sites prior to osimertinib. All patients in molecular analysis set underwent genetic testing at least three times during course of disease: before first EGFR-TKI treatment, pre-osimertinib and after osimertinib resistance. Samples for genetic testing before first EGFRTKI treatment were mostly tumor tissues $(43 / 49,87.8 \%)$; while, samples used for pre-osimertinib and after osimertinib resistance testing were both mostly plasma (34/49, $69.4 \%)$.

\section{Clinical outcomes}

Of 49 patients in the molecular analysis set, baseline preosimertinib molecular analysis showed that 30 patients had $\mathrm{T} 790 \mathrm{M}+/ 19 \mathrm{del}+, 17 \mathrm{had} \mathrm{T} 790 \mathrm{M}+/ \mathrm{L} 858 \mathrm{R}+$ and $2 \mathrm{had}$ T790M+/19del-/L858R-. The median PFS for osimertinib was 9.0 months $(95 \% \mathrm{CI} 8.2,9.8)$ and varied across genotypes. In patients with $\mathrm{T} 790 \mathrm{M}+/ 19 \mathrm{del}+$, the median PFS was 9.3 months (95\% CI 1.5, 17.1), which was significantly longer than patients with $\mathrm{T} 790 \mathrm{M}+/ \mathrm{L} 858 \mathrm{R}+$ (median: 8.5 months [95\% CI 6.3, 10.7]) $(P=0.005)$. A total of 45 patients were available for evaluation of the disease response in these two genotypes. The DCR was $100 \%(29 / 29)$ in patients with $\mathrm{T} 790 \mathrm{M}+/ 19 \mathrm{del}+$ and $93.8 \%(15 / 16)$ in patients with $\mathrm{T} 790 \mathrm{M}+/ \mathrm{L} 858 \mathrm{R}+(P=0.356)$.

In patients who developed resistance to osimertinib, possible genomic resistance mutations were identified in 27 of 49 patients in the molecular analysis set. Fourteen patients $(14 / 49,28.6 \%)$ acquired secondary EGFR C797S mutation, all in cis with the initial EGFR exon19del/L858R mutation and the T790M mutation. Other EGFR-dependent molecular modifications included C796S mutation (1/49, 2.0\%), G796S mutation $(1 / 49,2.0 \%)$, V802I mutation $(1 / 49,2.0 \%)$, V834L mutation (1/49, 2.0\%), E758D mutation (1/49, 2.0\%), G724S mutation $(1 / 49,2.0 \%)$ and EGFR amplification $(2 / 49,4.1 \%)$.
Table 1 Baseline patient demographic and clinical characteristics of the molecular analysis set

\begin{tabular}{|c|c|c|}
\hline \multirow[t]{2}{*}{ Characteristics } & \multicolumn{2}{|c|}{ Patients $(n=49)$} \\
\hline & No & $\%$ \\
\hline \multicolumn{3}{|l|}{ Age (years) } \\
\hline Median & 59 & \\
\hline Range & $42-84$ & \\
\hline \multicolumn{3}{|l|}{ Sex } \\
\hline Male & 19 & 38.8 \\
\hline Female & 30 & 61.2 \\
\hline \multicolumn{3}{|l|}{ ECOG PS } \\
\hline 0 & 27 & 55.1 \\
\hline 1 & 20 & 40.8 \\
\hline 2 & 2 & 4.1 \\
\hline \multicolumn{3}{|l|}{ Smoking status } \\
\hline Non-smoker & 38 & 77.6 \\
\hline Former/current smoker & 11 & 22.4 \\
\hline \multicolumn{3}{|l|}{ Histology } \\
\hline Adenocarcinoma & 48 & 98.0 \\
\hline Others & 1 & 2.0 \\
\hline \multicolumn{3}{|l|}{ Genotypes } \\
\hline \multicolumn{3}{|l|}{ T790M-positive } \\
\hline Exon 19del-positive & 30 & 61.2 \\
\hline L858R-positive & 17 & 34.7 \\
\hline Exon 19del/ L858R-negative & 2 & 4.1 \\
\hline \multicolumn{3}{|l|}{ Osimertinib treatment line } \\
\hline 2 nd & 25 & 51.0 \\
\hline$\geq 3 \mathrm{rd}$ & 24 & 49.0 \\
\hline \multicolumn{3}{|l|}{ Treatment history } \\
\hline Gefitinib & 25 & 51.0 \\
\hline Erlotinib & 10 & 20.4 \\
\hline Icotinib & 18 & 36.7 \\
\hline Afatinib & 1 & 2.0 \\
\hline Avitinib & 3 & 6.1 \\
\hline Chemotherapy & 25 & 51.0 \\
\hline \multicolumn{3}{|l|}{ Metastases } \\
\hline Lung & 38 & 77.6 \\
\hline Bone & 28 & 57.1 \\
\hline Pleural & 19 & 38.8 \\
\hline CNS & 18 & 36.7 \\
\hline Liver & 6 & 12.2 \\
\hline Adrenal gland & 6 & 12.2 \\
\hline
\end{tabular}

CNS central nervous system, ECOG PS Eastern Cooperative Oncology Group performance status

Non-EGFR modifications were identified in 10 patients $(10 / 49,20.4 \%)$ including activating mutations of PIK3CA $(1 / 49,2.0 \%), \operatorname{ALK}(1 / 49,2.0 \%), \operatorname{BRAF}(1 / 49,2.0 \%)$, KRAS $(2 / 49,4.1 \%)$, TP53 $(3 / 49,6.1 \%)$ and MET amplification $(3 / 49,6.1 \%)$. Nineteen patients had no new findings in osimertinib resistance testing except for pre-existing EGFR 
mutations pre-osimertinib, among whom 12 patients showed T790M-loss. Negative NGS results of plasma ctDNA samples were found in the remaining three patients. A summary of resistance mechanisms to osimertinib are shown in Fig. 2. Patients with an EGFR-dependent resistance mechanism more likely occurred in the base of exon 19del (53.3\% in exon19del vs $17.6 \%$ in L858R, $P=0.017$ ); while, alternative pathway activation showed a trend to present in the base of the L858R mutation (10.0\% in exon19del vs $23.5 \%$ in L858R, $P=0.409$ ).

T790M-loss was the most frequent phenomenon in the molecular analysis set, observed in $25(51.0 \%)$ patients and T790M-retain was found in 24 patients $(49.0 \%)$. We explored the prognosis difference between patients with T790M-retain and T790M-loss after progression with osimertinib therapy. Patients with T790M-retain showed a trend of longer PFS, but this was not statistically significant compared to patients with T790M-loss (median PFS, 9.3 months [95\% CI $4.9,13.7$ ] vs 7.8 months [95\% CI 5.4, $10.2], P=0.053)$. After disease progression, $29(59.2 \%)$ of 49 patients continued osimertinib with additional clinical benefit for a median period of 4.2 months (range 1.0-16.3), including 16 patients in the T790M-retain group and 13 patients in the T790M-loss group. The TD of osimertinib was 20.2 months (95\% CI 14.3, 26.1) for the T790M-retain group, which was significantly longer than 10.5 months (95\% CI 5.8, 15.3) of patients with T790M-loss $(P=0.026)$. We also explored clinical outcome differences between patients who had EGFR-dependent resistance mechanisms and patients with alternative pathway activation. Alternative pathway activation was identified in seven patients including PIK3CA, BRAF and KRAS mutations and MET amplification. Compared with T790M-retain, alternative pathway activation was more likely to found in T790M-loss patients $(8.3 \%$ vs $20.0 \%, P=0.448)$. Patients with alternative pathway activation after osimertinib resistance had shorter PFS and TD than patients with EGFR-dependent resistance mechanisms (median PFS, 8.2 months [95\% CI 3.6, 12.8] vs 13.5 months [95\% CI 5.5, 21.5], $P=0.012$; median TD,
9.5 months [95\% CI $6.2,12.8$ ] vs 16.6 months [95\% CI 10.4, 22.8], $P<0.001$ ) (Figs. 3, 4).

Outside the molecular analysis set, four patients with primary lung adenocarcinoma showed SCLC transformation after osimertinib resistance. Among the SCLC transformation population, three were men and heavy smokers, one was woman and a non-smoker. The original EGFR mutation in one patient was retained except for T790M in the plasma ctDNA sample. The other three patients did not receive gene testing as genotype transformation was detected. These four patients progressed on osimertinib with times that ranged from 1.4 to 5.6 months. The treatment for four SCLC transformation patients was a switch to etoposide/irinotecan plus platinum chemotherapy.

\section{Discussion}

Drug resistance represents a major challenge in targeted cancer therapy. Several studies have reported mechanisms of resistance to third-generation EGFR-TKI osimertinib including C797S mutation, EGFR amplification, bypass activation, phenotypic transformation and so on (Ortiz-Cuaran et al. 2016; Oxnard et al. 2018). Our data add to these findings, providing additional Chinese data setting, the associations of various molecular modifications, and clinical outcomes for possible resistance mechanisms.

Twenty-seven patients had putative genomic resistance mechanisms identified. We found the EGFR C797S mutation to be the most common resistance mechanism to osimertinib in our study, and all were concurrent in cis with T790M mutation. This mutation was detected in approximately $20-30 \%$ of osimertinib acquired resistance cases and was resistant to multiple EGFR-TKIs including gefitinib, erlotinib, afatinib and osimertinib when C797S performed in cis with a T790M mutation (Niederst et al. 2015; Thress et al. 2015; Sullivan and Planchard 2016). The combination of afatinib and cetuximab demonstrated promising clinical activity and a manageable safety profile
A

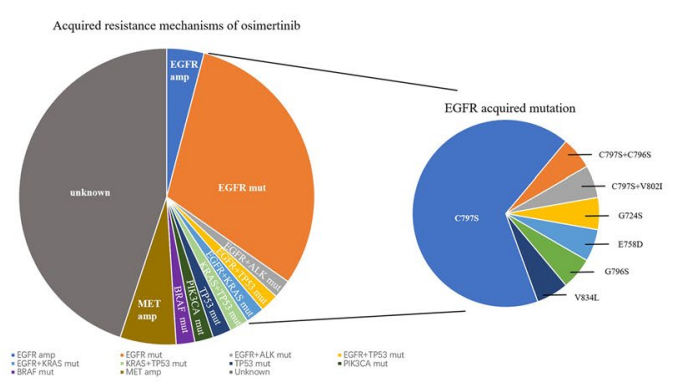

B

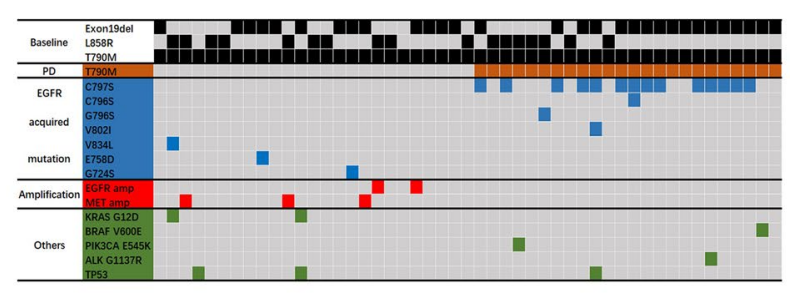

Fig. 2 Molecular modification after acquired resistance to osimertinib 
A PFS in patients with T790M-retain or T790M-loss

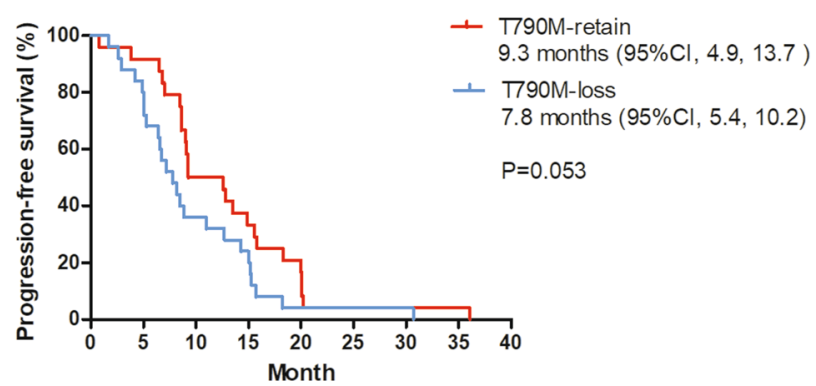

C TD in patients with T790M-retain or T790M-loss

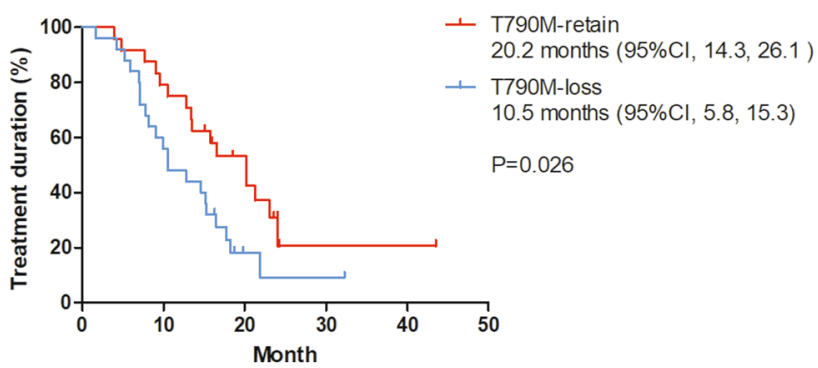

Fig. 3 PFS in patients with T790M-retain or T790M-loss after progression of osimertinib therapy (a), in patients with EGFR-dependent resistance mechanism or bypass activation after progression (b). TD in patients with T790M-retain or T790M-loss after progression of

in patients who developed acquired resistance to EGFRTKIs, regardless of the T790M mutation status (Janjigian et al. 2014), which may conquer such EGFR triple mutations. The research of treatment strategies for other EGFRdependent resistance mechanisms was lacking and needs further investigation. The combination of chemotherapy, bevacizumab and atezolizumab in the IMpower 150 study was promising for the EGFR mutated, prior EGFR-TKI failed patients (Reck et al. 2019). EGFR-independent resistance mechanisms were mainly bypass activation and mostly were mutually exclusive with the T790M mutation, suggesting the possibility of combination therapy. MET amplification is a common mechanism of resistance with an incidence of 10-30\% (Oxnard et al. 2018; Wang et al. 2018). The combination of EGFR-TKI and crizotinib was reported effective against the acquired MET amplification after progression of osimertinib therapy in the clinical setting (Wang et al. 2018). Activation of the RAS-MAPK pathway, such as KRAS mutation and BRAF mutation, was also reported. A combination of MEK or BRAF inhibitors may rescue such mechanism after resistance to osimertinib has developed (Eberlein et al. 2015; Ho et al. 2017). A negative NGS outcome after osimertinib resistance was detected in three patients in our study, which
B PFS in patients with EGFR-dependent resistance or bypass activation

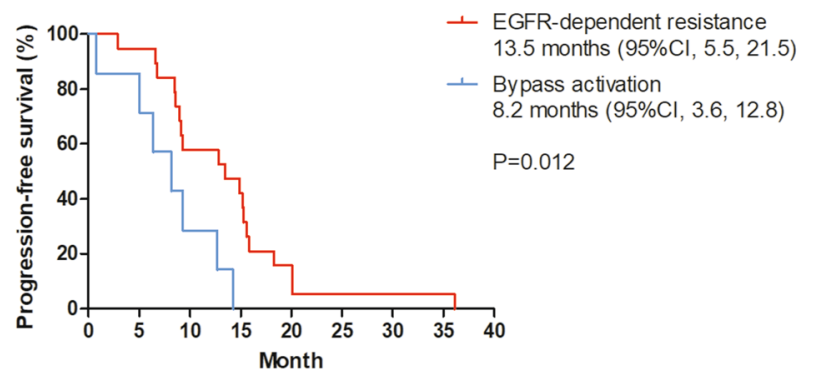

D TD in patients with EGFR-dependent resistance or bypass activation

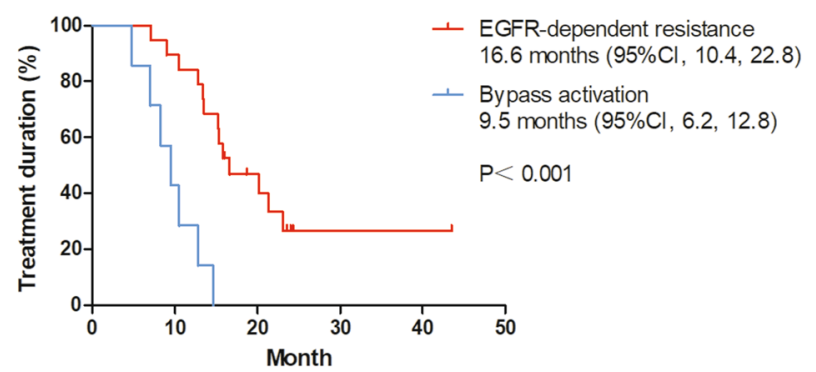

osimertinib (c), in patients with an EGFR-dependent resistance mechanism or bypass activation after progression (d). PFS progressionfree survival, $T D$ treatment duration, $C I$ confidence interval. Tick marks indicate censored observations

may result from the relatively low tumor burden, with low levels of ctDNA in plasma samples.

T790M-loss and T790M-retain were two basic modes at the time of PD. Loss of T790M was observed in over half of the patients in our study as previously reported (Oxnard et al. 2018), mostly concurrent with the development of bypass activation, and might be associated with earlier resistance to osimertinib. We found that T790M-retain patients had better clinical outcomes to osimertinib including PFS and TD than those with T790M-loss. As patients with T790M-loss are more likely to be associated with the development of bypass activation, the patient cohort with bypass activation in our study had similarly worse outcome than patients with an EGFR-dependent resistance mechanism. The association of bypass activation with the clinical outcome to osimertinib therapy has not previously been reported in detail. Yong $\mathrm{He}$ et al. analyzed the correlation of MET amplification and survival outcomes, and found that MET amplification was associated with shorter PFS and overall survival (OS) than in those patients without it after osimertinib progression (Wang et al. 2018). The clinical outcomes of bypass activation need further exploration.

Outside the molecular analysis set, four patients had SCLC transformation after osimertinib resistance. SCLC 
Fig. 4 Treatment duration in 49 patients in the molecular analysis set

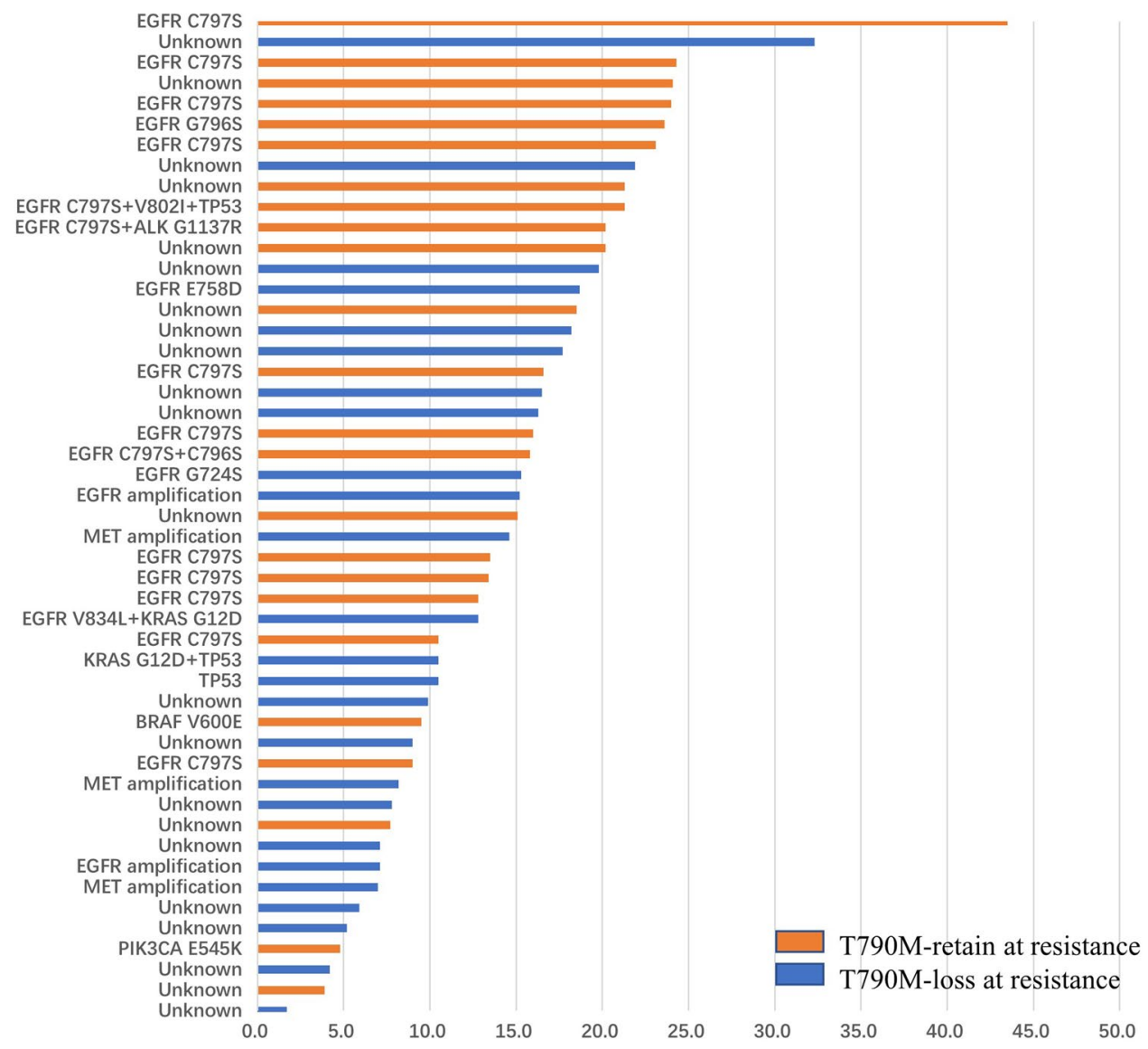

Treatment duration, months transformation has been reported as a mechanism of acquired resistance in $3 \sim 14 \%$ of patients progressing from first- to third-generation EGFR-TKIs (Sequist et al. 2011; $\mathrm{Yu}$ et al. 2013). After the confirmation of SCLC transformation, platinum-etoposide regimen is recommended (Marcoux et al. 2019).

There were several limitations to our study. First, the study was limited to a single center, its retrospective design and small sample size. Second, molecular data of both tissue and plasma samples, whichever were available, were collected for molecular analysis, which may bias the results of the molecular modifications. Future tissue-based analyses are needed to provide a more comprehensive profile of the mechanisms underlying resistance to osimertinib.

In conclusion, molecular re-analysis after osimertinib failure has significant clinical utility for guiding personalized subsequent treatment selection. Further studies on novel or combination therapy are needed to overcome the resistance to osimertinib.

Acknowledgements The authors would like to thank all study patients and their family members.
Author contributions JL designed the study, YM collected the data, performed analysis and wrote the manuscript, PX collected the data and revised the manuscript, $\mathrm{XH}, \mathrm{XH}, \mathrm{YW}, \mathrm{TL}, \mathrm{JZ}$ and $\mathrm{ZX}$ collected the data.

Data availability The data are not publicly available due to privacy and ethical restrictions.

\section{Compliance with ethical standards}

Conflict of interest The authors have no conflict of interest.

Ethical approval This study was conducted according to the principles of the Declaration of Helsinki, and approved by the Ethics Committee of the National Cancer Center/Cancer Hospital, Chinese Academy of Medical Sciences and Peking Union Medical College.

Open Access This article is licensed under a Creative Commons Attribution 4.0 International License, which permits use, sharing, adaptation, distribution and reproduction in any medium or format, as long as you give appropriate credit to the original author(s) and the source, provide a link to the Creative Commons licence, and indicate if changes were made. The images or other third party material in this article are included in the article's Creative Commons licence, unless indicated otherwise in a credit line to the material. If material is not included in the article's Creative Commons licence and your intended use is not 
permitted by statutory regulation or exceeds the permitted use, you will need to obtain permission directly from the copyright holder. To view a copy of this licence, visit http://creativecommons.org/licenses/by/4.0/.

\section{References}

Cross DA et al (2014) AZD9291, an irreversible EGFR TKI, overcomes T790M-mediated resistance to EGFR inhibitors in lung cancer. Cancer Discov 4:1046-1061

Eberlein CA et al (2015) Acquired resistance to the mutant-selective EGFR inhibitor AZD9291 is associated with increased dependence on RAS signaling in preclinical models. Cancer Res 75:2489-2500

Ho CC, Liao WY, Lin CA, Shih JY, Yu CJ, Chih-Hsin YJ (2017) Acquired BRAF V600E mutation as resistant mechanism after treatment with osimertinib. J Thorac Oncol 12:567-572

Janjigian YY et al (2014) Dual inhibition of EGFR with afatinib and cetuximab in kinase inhibitor-resistant EGFR-mutant lung cancer with and without T790M mutations. Cancer Discov 4:1036-1045

Lee K et al (2019) Repeat biopsy procedures and T790M rates after afatinib, gefitinib, or erlotinib therapy in patients with lung cancer. Lung Cancer 130:87-92

Marcoux N et al (2019) EGFR-mutant adenocarcinomas that transform to small-cell lung cancer and other neuroendocrine carcinomas: clinical outcomes. J Clin Oncol 37:278-285

Mok TS et al (2009) Gefitinib or carboplatin-paclitaxel in pulmonary adenocarcinoma. N Engl J Med 361:947-957

Mok TS et al (2017) Osimertinib or platinum-pemetrexed in EGFR T790M-positive lung cancer. N Engl J Med 376:629-640

Niederst MJ et al (2015) The allelic context of the C797S mutation acquired upon treatment with third-generation EGFR inhibitors impacts sensitivity to subsequent treatment strategies. Clin Cancer Res 21:3924-3933

Ortiz-Cuaran S et al (2016) Heterogeneous mechanisms of primary and acquired resistance to third-generation EGFR inhibitors. Clin Cancer Res 22:4837-4847

Oxnard GR et al (2018) Assessment of resistance mechanisms and clinical implications in patients with EGFR T790M-positive lung cancer and acquired resistance to osimertinib. JAMA Oncol $4: 1527-1534$
Reck M et al (2019) Atezolizumab plus bevacizumab and chemotherapy in non-small-cell lung cancer (IMpower150): key subgroup analyses of patients with EGFR mutations or baseline liver metastases in a randomised, open-label phase 3 trial. Lancet Respir Med 7:387-401

Rosell R et al (2012) Erlotinib versus standard chemotherapy as first-line treatment for European patients with advanced EGFR mutation-positive non-small-cell lung cancer (EURTAC): a multicentre, open-label, randomised phase 3 trial. Lancet Oncol 13:239-246

Sequist LV et al (2011) Genotypic and histological evolution of lung cancers acquiring resistance to EGFR inhibitors. Sci Transl Med $3: 26 \mathrm{r}-75 \mathrm{r}$

Sequist LV et al (2013) Phase III study of afatinib or cisplatin plus pemetrexed in patients with metastatic lung adenocarcinoma with EGFR mutations. J Clin Oncol 31:3327-3334

Shi Y et al (2014) A prospective, molecular epidemiology study of EGFR mutations in Asian patients with advanced non-small-cell lung cancer of adenocarcinoma histology (PIONEER). J Thorac Oncol 9:154-162

Siegel RL, Miller KD, Jemal A (2015) Cancer statistics, 2015. CA Cancer J Clin 65:5-29

Sullivan I, Planchard D (2016) Osimertinib in the treatment of patients with epidermal growth factor receptor T790M mutation-positive metastatic non-small cell lung cancer: clinical trial evidence and experience. Ther Adv Respir Dis 10:549-565

Thress KS et al (2015) Acquired EGFR C797S mutation mediates resistance to AZD9291 in non-small cell lung cancer harboring EGFR T790M. Nat Med 21:560-562

Wang Y, Li L, Han R, Jiao L, Zheng J, He Y (2018) Clinical analysis by next-generation sequencing for NSCLC patients with MET amplification resistant to osimertinib. Lung Cancer 118:105-110

Yu HA et al (2013) Analysis of tumor specimens at the time of acquired resistance to EGFR-TKI therapy in 155 patients with EGFRmutant lung cancers. Clin Cancer Res 19:2240-2247

Publisher's Note Springer Nature remains neutral with regard to jurisdictional claims in published maps and institutional affiliations. 